\title{
Chaotic dynamics of a Bose-Einstein condensate in a double-well trap
}

\author{
P Coullet and N Vandenberghe \\ Institut Non Linéaire de Nice, CNRS-UNSA, 1361 Route des Lucioles, 06560, Valbonne, France
}

Received 21 February 2002

Published 13 March 2002

Online at stacks.iop.org/JPhysB/35/1593

\begin{abstract}
The dynamics of Bose-Einstein condensates in a double-well magnetic trap is studied using the Gross-Pitaevskii equation (GPE) and a two-mode approximation. The self-trapping instability occurs when a sufficiently high potential barrier is formed in the centre of the trap. Close to the instability threshold, the GPE is reduced to a simple amplitude equation. In the case where weakly dissipative effects are taken into account, the condensate exhibits aperiodic oscillations which can be described by the Lorenz thermal convection model.
\end{abstract}

\section{Introduction}

At low enough temperatures, Bose-Einstein condensates (BECs) of trapped gases [1-3] can be described by the Gross-Pitaevskii equation (GPE) [4]:

$$
\mathrm{i} \hbar \partial_{t} \Psi=-\frac{\hbar^{2} \vec{\nabla}^{2}}{2 m} \Psi+g|\Psi|^{2} \Psi+V(\vec{r}) \Psi,
$$

where $\Psi(\vec{r}, t)$ is the condensate wavefunction, $g=4 \pi \hbar^{2} a / m$ and $m$ is the atomic mass. The role of interactions between atoms is accounted for by the s-wave scattering length $a$ which can be positive (for repulsive species: $\mathrm{Na}, \mathrm{Rb}, \mathrm{H}$ ) or negative (for attractive species: $\mathrm{Li}$ ). It is possible to vary $a$ (and to reverse its sign) during an experiment by using magnetic-fieldinduced Feshbach resonance [5].

In what follows, we will consider a thin cigar-shaped condensate. If the radial confinement is strong enough, we can neglect the transversal dynamic and the condensate can be described by a one-dimensional version of the GPE. In one dimension, the GPE can be rescaled to the non-dimensional form

$$
\mathrm{i} \partial_{t} \Psi=-\partial_{x x}^{2} \Psi+g|\Psi|^{2} \Psi+V(x) \Psi,
$$

where $x$ is measured in units of the healing length $\xi=(4 \pi a n)^{-1 / 2}, t$ is rescaled by the trapping frequency $\omega_{t}=4 \pi \hbar a n / m$ and $\Psi$ is normalized by the square root of the density, $n$. This equation will be referred to as the nonlinear Schrödinger equation (NLSE) although it includes the additional potential term. 


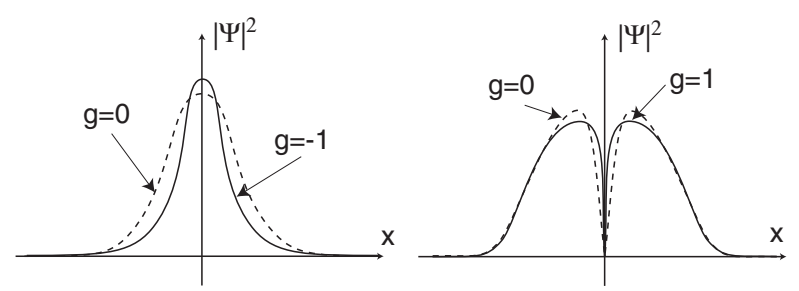

Figure 1. Sketch of the effect of nonlinearity on the ground state and the black soliton.

Stationary solutions of the NLSE are written as $\Psi_{s}=u_{\mathrm{s}}(x) \mathrm{e}^{-\mathrm{i} \omega_{s} t}$. The shape of a condensate formed in a harmonic magnetic trap is the ground state of the NLSE. When nonlinear effects can be neglected $(g=0)$, the ground state is Gaussian. For a fixed number of atoms in the condensate, a negative scattering length tends to increase the density and decrease the spatial extension of the condensate. A positive scattering length has the opposite effect. In addition to the ground state, we will also consider the first excited state [6] (also called a black soliton or kink). This corresponds to the stationary solution with a single node. The effect of nonlinearity due to interactions is to decrease the characteristic size of the ground state (respectively, the first excited state) in the case of attractive interactions (respectively repulsive interactions). In what follows, this effect will be referred to as nonlinear localization (figure 1).

A widely tunable double-well potential has been realized experimentally by focusing a far-off-resonant laser sheet into the centre of the magnetic trap [7]. The laser power and the width of the sheet are widely tunable. The barrier, which tends to repel atoms from the centre of the trap, competes with the nonlinear localization. This competition gives rise to the self-trapping instability [8-10].

The NLSE describes ideal BECs that conserve mass and energy. Condensates, as observed experimentally, are weakly dissipative and decay over time [11]. In what follows, when irreversible effects are considered, we will also assume the existence of a process that continuously brings the atom into the condensed state ${ }^{1}$. Although there is no completely established theory of such dissipative effects, we will consider a weakly dissipative version of the Gross-Pitaevskii model in order to describe the dynamics of BECs near the self-trapping instability.

The goal of this paper is to describe the self-trapping instability in the reversible case and when small irreversible effects are taken into account. Reduced-dimensional amplitude equations and direct numerical simulations of the NLSE are used to explore the dynamics. In particular, it is shown that the quasi-reversible dynamic of the BECs near self-trapping can be described by the Lorenz equations [12].

This paper is organized as follows: for pedagogical reasons, in the first part we discuss the two-mode model while the full analysis of the GPE is performed in the second part. In both parts reversible and weakly irreversible dynamics are discussed.

\section{The two-mode model}

\subsection{Weakly interacting Bose-Einstein condensate}

The two-mode model for a BEC $[9,13]$ in a well separated two-well trap reads

$$
\mathrm{i} \dot{A_{1}}=g\left|A_{1}\right|^{2} A_{1}-\kappa A_{2},
$$

1 Although we are not aware of the existence of such a process in actual experiments, it will be an important step for the realization of an atom laser. 


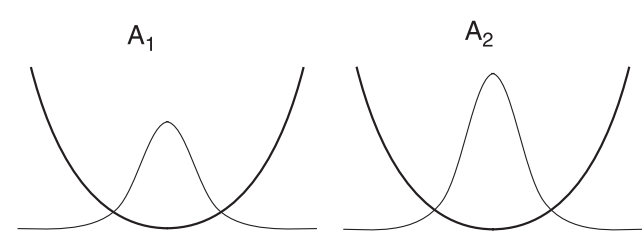

Figure 2. Sketch of a BEC in a double-well trap.

$$
\mathrm{i} \dot{A_{2}}=g\left|A_{2}\right|^{2} A_{2}-\kappa A_{1}
$$

$A_{i}$ is the amplitude of the linear ground state in each well (figure 2), $\left|A_{i}\right|^{2}$ measures the number of atoms in each well and the nonlinear eigenfrequencies $g\left|A_{i}\right|^{2}$ represent the effect of atomic interactions in the BEC. The parameter $g$ can be positive (repulsive species) or negative (attractive species). The tunnelling term $\kappa A_{j}$ describes the weak interaction between the two wells.

The two-mode model is accurate in describing a BEC with a weak interatomic interaction, i.e. with a low number of atoms or a low scattering length and a strong potential barrier such that the interaction between the atoms in each well is weak [14]. It is a natural nonlinear extension of the two-mode model that describes the ammonia maser [15]. We propose, in appendix A, a simple derivation of this model.

The parameter $g$ can be rescaled to +1 for repulsive species or -1 for attractive species.

\subsection{Stationary solutions of the two-mode model}

The first step is to compute the stationary solutions of the two-mode model. We write

$$
A_{1}=\rho_{1} \mathrm{e}^{-\mathrm{i} \varphi_{1}}, \quad A_{2}=\rho_{2} \mathrm{e}^{-\mathrm{i} \varphi_{2}},
$$

where $\rho_{i}$ are positive real numbers and $\varphi_{i}$ are real numbers. Using the variables $m=\rho_{1}^{2}+\rho_{2}^{2}$, $\delta=\rho_{2}^{2}-\rho_{1}^{2}$ and $\varphi=\varphi_{2}-\varphi_{1}$ we have the equations

$$
\begin{aligned}
& \dot{m}=0, \\
& \dot{\delta}=-2 \kappa \sqrt{m^{2}-\delta^{2}} \sin \varphi, \\
& \dot{\varphi}=g \delta+\kappa \frac{2 \delta}{\sqrt{m^{2}-\delta^{2}}} \cos \varphi .
\end{aligned}
$$

The quantity $m$ is conserved. This follows from the conservation of mass of the condensate.

From equation (7), it follows that the stationary solutions are either in phase $(\varphi=0)$ or out of phase $(\varphi=\pi)$. There are two obvious stationary solutions with $\delta=0$ and either $\varphi=0$ or $\pi$. Both solutions exist for any values of $g$ and $m$. They are the nonlinear continuations of the classical linear in-phase and out-of-phase solutions.

However, the interesting feature of the nonlinear two-mode model is that there exists trapped asymmetric solutions for which $\delta \neq 0$. It follows from equation (8) that

$$
g \sqrt{m^{2}-\delta^{2}}+2 \kappa \xi=0,
$$

where $\xi=\cos \varphi= \pm 1$.

Equation (9) shows that trapped solutions exist only when $g$ and $\xi$ have opposite signs. That means that the trapped solution is in phase for the attractive condensate $(g<0)$ and out of phase for the repulsive condensate $(g>0)$.

Trapped solutions are a result of the competition between nonlinearity and dispersion induced by tunnelling. They exist if

$$
|g| m>2 \kappa .
$$




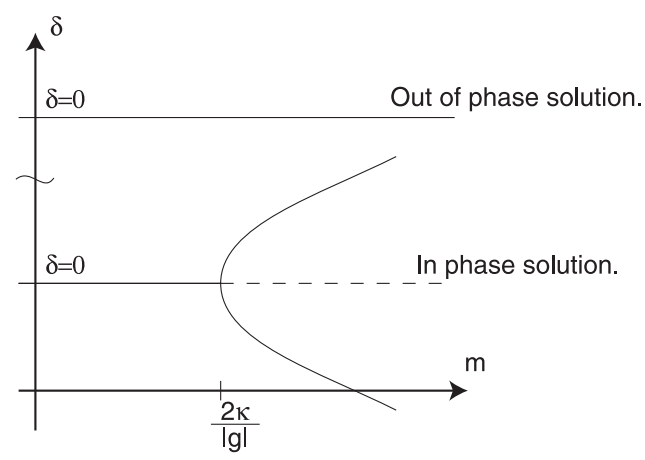

Figure 3. Bifurcation diagram of the two-mode model of an attractive condensate when $m$ increases.

The existence of such non-symmetric states comes from the quasi-classical approximation that underlies the derivation of the NLSE [16]. They actually represent long-lived metastable states such as have been observed in the vibration of dihalomethane molecules [8], for example.

A small amount of energy given initially to one of the oscillators will tunnel to the other one through resonant interaction. Self-trapping occurs because larger intensities lead to nonlinear oscillations far from resonance. A simple classical interpretation is the following: equations (3) and (4) can be seen as the amplitude equations of the small oscillations of two weakly coupled nonlinear oscillators (appendix A).

\subsection{The self-trapping instability}

As shown in [10] the existence of the trapped solution is the result of a symmetry breaking bifurcation (pitchfork bifurcation) of the in-phase solution (respectively the out of phase solution) for the attractive (respectively repulsive) condensate. The bifurcation diagram presented in figure 3 shows the equilibrium position as a function of the bifurcation parameter.

In order to capture the dynamic of the condensate near self-trapping, the two-mode model is reduced to simpler amplitude equations using standard asymptotic techniques. We will focus on the instability of the in-phase solution $(\varphi=0)$ that occurs when the condensate is attractive, i.e. $g<0$. We rescale $\delta^{\prime}=-g \delta$ and $m^{\prime}=-g m$ such that $g$ no longer appears in equation (8) and is replaced by -1 . The ' will be omitted.

Let us introduce $m_{0}$ as a reference mass of the condensate and write $m=m_{0}+\sigma$.

The in-phase solution is then $\varphi=0, \delta=0, \sigma=0$ and its stability is given by the linearized equation of the matrix

$$
\frac{\mathrm{d}}{\mathrm{d} t}\left(\begin{array}{c}
\sigma \\
\delta \\
\varphi
\end{array}\right)=\left(\begin{array}{ccc}
0 & 0 & 0 \\
0 & 0 & -2 \kappa m_{0} \\
0 & -1+2 \kappa / m_{0} & 0
\end{array}\right)\left(\begin{array}{c}
\sigma \\
\delta \\
\varphi
\end{array}\right) .
$$

The instability occurs when the two non-trivial eigenvalues of this matrix vanish, i.e. for $\kappa=\kappa_{c}=m_{0} / 2$ and the in-phase solution loses its stability when $\kappa<\kappa_{c}$ since decreasing $\kappa$ increases the potential barrier.

We introduce the small parameter $\mu=\left(\kappa_{c}-\kappa\right) / \kappa_{c}$ which measures the distance from the instability threshold.

As usual, close to a bifurcation pitchfork, the variables scale as $\sigma \sim \mathrm{O}(\mu), \delta \sim \mathrm{O}(\sqrt{|\mu|})$ and $\phi \sim \mathrm{O}(\mu)$ and they depend on a slow timescale $\mathrm{d} / \mathrm{d} t \sim \mathrm{O}(\sqrt{|\mu|})$. At leading order we get 


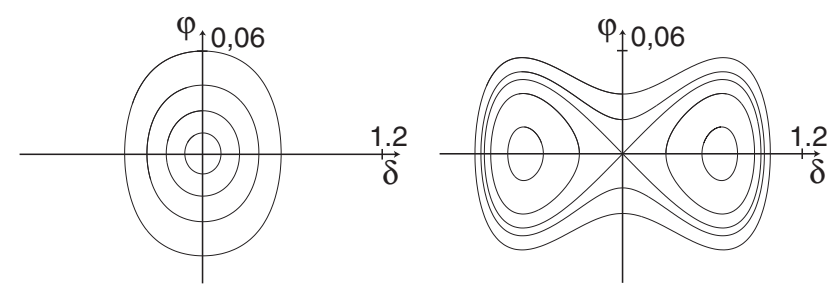

Figure 4. Phase space for equations (7) and (8) for $\kappa=1.15, m=2.2$ (left) and $m=2.4$ (right) .

$$
\begin{aligned}
& \dot{\sigma}=0, \\
& \ddot{\delta}=\mu m_{0}^{2} \delta+m_{0} \sigma \delta-\frac{1}{2} \delta^{3},
\end{aligned}
$$

or, with $Q=m_{0} \sigma, X=\delta / \sqrt{2}$ and $\tilde{\mu}=\mu m_{0}^{2}$

$$
\dot{Q}=0 \text {, }
$$$$
\ddot{X}=\tilde{\mu} X+Q X-X^{3} .
$$

These two equations describe the reversible self-trapping bifurcation. When the parameter $\mu$ grows, i.e. when the potential barrier increases, the instability threshold is reached and the phase plane of the dynamical system exhibits non-symmetric oscillations. Increasing the mass of the condensate can also lead to the same instability. On the phase plane of equations (7) and (8) (figure 4), for $\mu>0$, we can identify the equilibrium points (or stationary solutions), the non-symmetric oscillations and large symmetric oscillations. These two types of behaviour are separated by a double homoclinic loop which acts as a separatrix between symmetric and non-symmetric oscillations. The oscillations have been described in terms of the Jacobian elliptic function [13].

\subsection{Quasi-reversible two-mode model}

Irreversible effects (like two- and three-body recombinations, interactions with the thermal cloud, ...) eject atoms from the condensate. In order to compensate these dissipative effects, we also have to consider the existence of a pump. The two-mode model can be generalized by adding small terms which explicitly break time-reversal of the initial equations $\left(t \rightarrow-t, A_{i} \rightarrow \bar{A}_{i}, i=1,2\right)$. Thus

$$
\begin{aligned}
& \mathrm{i} \dot{A_{1}}=g\left|A_{1}\right|^{2} A_{1}-\kappa A_{2}+\mathrm{i} \lambda\left(\alpha A_{1}-\beta_{2}\left|A_{1}\right|^{2} A_{1}+\gamma A_{2}\right), \\
& \mathrm{i} \dot{A_{2}}=g\left|A_{2}\right|^{2} A_{2}-\kappa A_{1}+\mathrm{i} \lambda\left(\alpha A_{1}-\beta_{2}\left|A_{1}\right|^{2} A_{1}+\gamma A_{2}\right) .
\end{aligned}
$$

$\lambda>0$ is a small parameter which measures the irreversible effects, $\alpha>0$ models the injection process, $\beta_{2}>0$ measures the losses due to two-body recombinations ${ }^{2}$ and $\gamma>0$ measures losses that follow from diffusion and inhomogeneous dissipation processes.

The main issue under discussion is how these small irreversible terms modify the dynamics close to the self-trapping bifurcation. From equations (16) and (17), we can deduce the equations for the mass $m$, the population imbalance $\delta$ and the phase difference $\varphi$

$$
\begin{aligned}
& \dot{m}=2 \lambda\left(\alpha m-\beta \frac{m^{2}+\delta^{2}}{2}+\gamma \sqrt{m^{2}-\delta^{2}} \cos \varphi\right), \\
& \dot{\delta}=-2 \kappa \sqrt{m^{2}-\delta^{2}} \sin \varphi+2 \lambda(\alpha \delta-\beta m \delta), \\
& \dot{\varphi}=-\delta+2 \kappa \frac{\delta}{\sqrt{m^{2}-\delta^{2}}} \cos \varphi-\lambda \gamma\left(\frac{2 m}{\sqrt{m^{2}-\delta^{2}}} \sin \varphi\right) .
\end{aligned}
$$

2 A term $\beta_{3}|A|^{4} A$, taking into account three-body recombination, could be included without introducing fundamental differences in our analysis. 

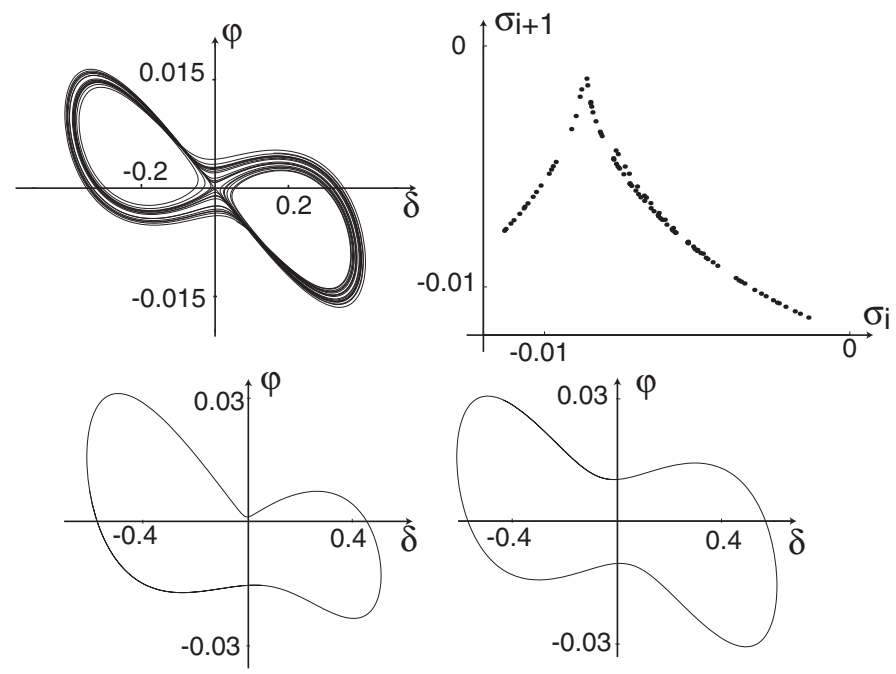

Figure 5. Projection of the phase space for equations (18)-(20). $m_{0}=2.4, g=-1, \epsilon=0.05$, $\alpha=1, \beta=1, \gamma=0.2$. (a) For $\kappa=1.18$ Lorenz attractor, and in (b) the mapping of the successive maxima of $\sigma$. $(c) \kappa=1.16$ non-symmetric limit cycle, $(d) \kappa=1.15$ symmetric limit cycle.

Equation (18) sets the reference mass. Therefore, we choose $m_{0}=2(\alpha+\gamma) / \beta$. The in-phase solution then reads $m=m_{0}, \delta=0$ and $\varphi=0$. In what follows we will assume $(\lambda \sim \mathrm{O}(\sqrt{|\mu|}))$ in order to keep the irreversible effects in the amplitude equation. With the same variables as in equations (14) and (15) we get

$$
\begin{aligned}
& \dot{Q}=-v_{Q} Q-\eta X^{2}, \\
& \ddot{X}=(\mu-\hat{\mu}) X-X^{3}+Q X-v_{X} \dot{X},
\end{aligned}
$$

where $\hat{\mu}=4 \lambda^{2} \gamma(\alpha+2 \gamma), v_{Q}=2 \lambda(\alpha+\gamma), v_{X}=2 \lambda(\alpha+3 \gamma), \eta=\lambda(4 \alpha+6 \gamma)$.

The stimulated loss $\eta X^{2}$ in equation (21) is at the origin of the most striking difference between the reversible and the quasi-reversible cases. Whenever the condensate is located nonsymmetrically in the trap, it is more focused that when it is in the centre of the trap. Therefore the losses due to two-body recombinations are stronger and so the mass of the condensate decreases. Since equations (21) and (22) are fully three dimensional, the coupling term induces very rich dynamics. As shown, for example, in [17], equations (21) and (22) are equivalent to the Lorenz equations that were first obtained in the context of thermal convection [12].

Different behaviours are possible depending on the values of the various parameters. Some typical behaviour of the original equations (18)-(20) are shown in figure 5.

\section{The nonlinear Schrödinger equation}

\subsection{Stationary solutions of the nonlinear Schrödinger equation}

In this part, we use the one-dimensional NLSE (2) to study the dynamics of the condensate when damping and finite-temperature effects are neglected. First we study the stationary solutions of the NLSE. To find a stationary solution, we have to fix the mass of the condensate $M=\int_{-\infty}^{+\infty}|\Psi|^{2} \mathrm{~d} x=1$. The potential that takes into account the combination of the magnetic trap and of the laser sheet can be written as [7] 

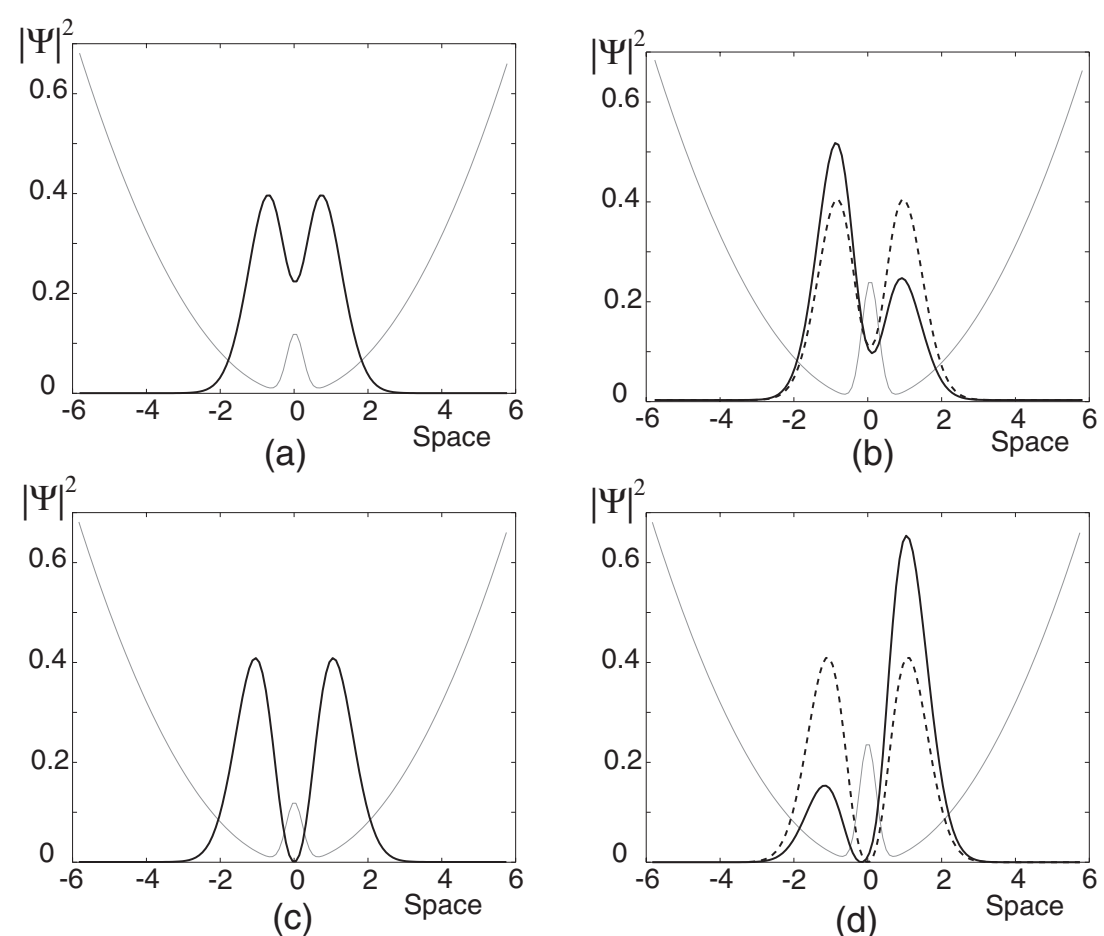

Figure 6. Some stationary solutions of the NLSE (equation (2)). (a) The ground state for $g=-1$ and $b=6$. (b) The symmetric unstable ground state (dotted curve) and one of the two nonsymmetric stable solution (plain curve) for $g=-1$ and $b=12$. (c) The kink state for $g=1$ and $b=6$. (d) The symmetric unstable kink state (dotted curve) and one of the two non-symmetric stable solutions (plain curve) for $g=1$ and $b=12$.

$$
V(x)=x^{2}+b \mathrm{e}^{-c x^{2}} \text {. }
$$

We numerically solve the nonlinear eigenvalue problem and find $\omega_{\mathrm{s}}$ and $u_{\mathrm{s}}$ such that

$$
\omega_{\mathrm{s}} u_{\mathrm{s}}=V(x) u_{\mathrm{s}}-\partial_{x x}^{2} u_{\mathrm{s}}+g u_{\mathrm{s}}^{3}
$$

where $\Psi_{s}(x, t)=u_{\mathrm{s}}(x) \mathrm{e}^{-\mathrm{i} \omega_{\mathrm{s}} t}$. A Crank-Nicholson scheme in time [18] is used. Some stationary solutions for the ground state and the dark soliton state are shown in figure 6.

Once more, it is interesting to look for non-symmetric solutions. For a large enough barrier height such stationary solutions can be found. Some examples of non-symmetric solutions are shown in figure 6. They arise from the self-trapping instability.

\subsection{The self-trapping instability}

The discussion given in the section 2 as well as the existence of non-symmetric solutions suggests the occurrence of a pitchfork bifurcation for the stationary solution. The self-trapping instability can be started by the potential bump increase and there exists a critical value $b_{c}$ of parameter $b$ for which the symmetric solution loses stability. We performed a numerical stability analysis of the solution $u_{\mathrm{s}}$ when $b$ varies. It reveals that:

- when $b$ is small, the ground state and the kink state are both stable,

- if $g$ is negative, when $b$ increases, the ground state becomes unstable for $b=b_{c}\left(b_{c}=9.14\right.$ for the values of figure 7); the kink state remains stable; 


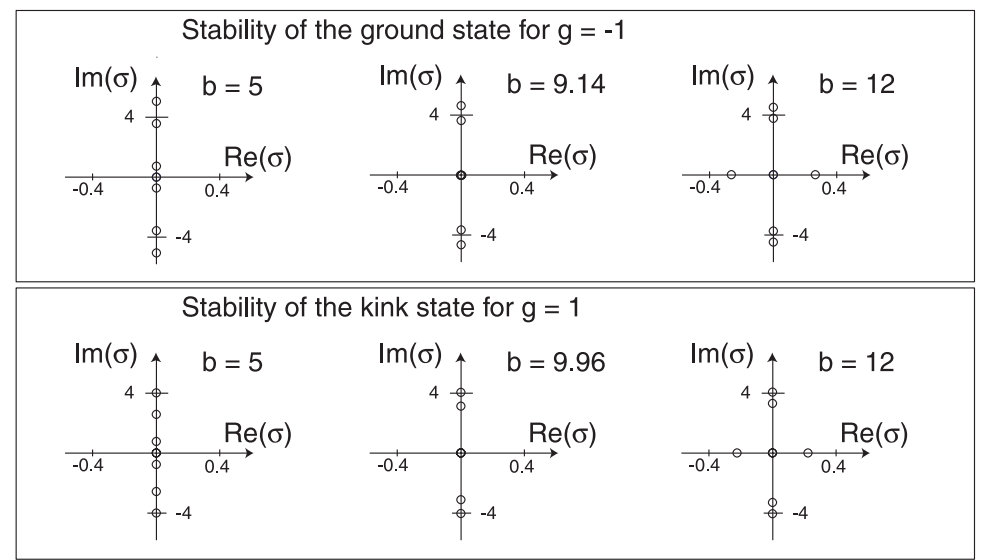

Figure 7. The motion of the eigenvalues of $\mathbb{L}$ when $b$ increases for the first mode when $g=-1, c=10$ (up) and for the second mode when $g=1, c=10$ (down).

- if $g$ is positive, when $b$ increases, the kink state becomes unstable for $b=b_{c}\left(b_{c}=9.96\right.$ for the values presented in figure 7); the ground state remains stable.

The linear operator associated with the linear evolution of a perturbation of $u_{\mathrm{s}}$ is $\mathbb{L}=$ $-\omega_{\mathrm{s}}-\partial_{x x}^{2}+V(x)+g u_{\mathrm{s}}^{2}+2 g u_{\mathrm{s}}^{2} \mathfrak{c} \mathfrak{j}$, where $\mathfrak{c j}$ denotes the conjugation of a complex number. The spectrum of $\mathbb{L}$ is plotted in figure 7 and clearly exhibits a reversible static bifurcation (the eigenfrequencies pass through zero). As we will see later, the symmetry of the eigenmodes is the signature of a pitchfork bifurcation.

We define the bifurcation parameter as

$$
\epsilon \mu=b-b_{c},
$$

where $\epsilon$ is a small parameter that measures the distance from the instability threshold and $\mu$ is a parameter a priori of order 1 that can be positive or negative.

To catch the dynamic close to the self-trapping we perturb the static solution and write

$$
\psi(x, t)=\left(u_{\mathrm{s}}(x)+u(x, t)\right) \mathrm{e}^{-\mathrm{i} \omega_{\mathrm{s}} t} \mathrm{e}^{-\mathrm{i} \varphi(x, t)},
$$

where $u(x, t)$ and $\varphi(x, t)$ are small and vary slowly in time. More precisely, we will use the standard scaling associated with a reversible pitchfork bifurcation

$$
\begin{aligned}
& u(x, t)=\sqrt{\epsilon} u_{(1)}(x, \tau)+\epsilon u_{(2)}(x, \tau)+\epsilon^{3 / 2} u_{(3)}(x, \tau)+\cdots, \\
& \varphi(x, t)=\sqrt{\epsilon} \varphi_{(1)}(x, \tau)+\epsilon \varphi_{(2)}(x, \tau)+\epsilon^{3 / 2} \varphi_{(3)}(x, \tau)+\cdots, \\
& V(x)=\left.V\right|_{0}(x)+\left.\epsilon \mu V\right|_{1}(x),
\end{aligned}
$$

where $\tau=\sqrt{\epsilon} t$ is the slow timescale and $\left.V\right|_{0}(x)=x^{2}+b_{c} \mathrm{e}^{-c x^{2}}$.

At $\operatorname{order} \epsilon^{0}$, we get the equation for the stationary solution

$$
\omega_{\mathrm{s}} u_{\mathrm{s}}=-\partial_{x x}^{2} u_{\mathrm{s}}+g u_{\mathrm{s}}^{3}+\left.V\right|_{0} u_{\mathrm{s}} .
$$

where $u_{\mathrm{s}}$ is either even (ground state) or odd (kink state).

At order $\sqrt{\epsilon}$, we have the eigenvalue problem

$$
\begin{array}{ll}
0=\mathcal{L}_{\varphi} u_{(1)} & \text { with } \mathcal{L}_{\varphi}=-\omega_{\mathrm{s}}-\partial_{x x}^{2}+3 g u_{\mathrm{s}}^{2}+\left.V\right|_{0}, \\
0=\mathcal{L}_{u} \varphi_{(1)} & \text { with } \mathcal{L}_{u}=2 \partial_{x} u_{\mathrm{s}} \partial_{x}+u_{\mathrm{s}} \partial_{x x}^{2} .
\end{array}
$$



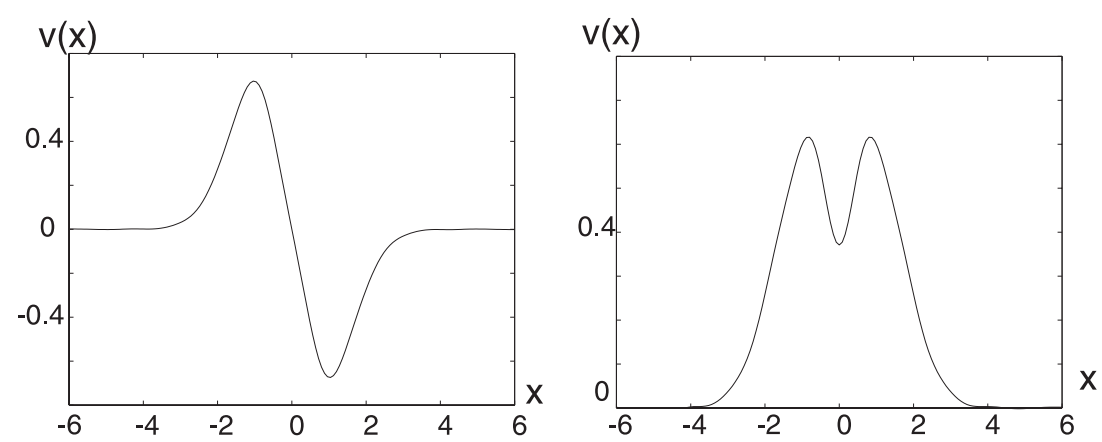

Figure 8. The eigenvectors $v$ for the bifurcation of the ground state $(g=-1, b=9.14$, $c=10)$ (left) and the kink state $(g=1, b=9.96, c=10)$ (right).

The linear operator $\mathcal{L}_{u}$ has an obvious zero eigenvalue associated with the eigenvector $\varphi_{(1)}=1$ (or any constant). This neutral mode arises from the phase invariance of the NLSE $\left(\Psi \rightarrow \Psi \mathrm{e}^{\mathrm{i} \Theta}\right)$.

Equation (31) determines the value of $b_{c}$. The operator $\mathcal{L}_{\varphi}$ has a zero eigenvalue for $b=b_{c}$, where $b_{c}$ is the value of $b$ at the onset of instability. Thus, for the particular value $b_{c}$, there is a double-zero eigenvalue and there exists a vector $v$ such that

$$
\mathcal{L}_{\varphi} v=0 .
$$

$v$ has the parity opposite ${ }^{3}$ to $u_{\mathrm{s}}$ (we note $v \oslash u_{\mathrm{s}}$ ). Two examples of eigenvectors $v$ are plotted in figure 8. It is also worth noting that $\mathcal{L}_{\varphi}$ is self-adjoint for the inner product $\langle a, b\rangle=\int_{-\infty}^{+\infty} a b \mathrm{~d} x$.

The first terms of the asymptotic development can be given as

$$
\varphi_{(1)}=\alpha(\tau) \quad \text { and } \quad u_{(1)}=A(\tau) v(x) .
$$

We can continue the asymptotic expansion to catch the weakly nonlinear dynamic close to the self-trapping bifurcation.

At order $\epsilon$, we have two equations for $u_{(2)}$ and $\varphi_{(2)}$ :

$$
\begin{aligned}
& u_{\mathrm{s}} \dot{\alpha}=\mathcal{L}_{\varphi} u_{(2)}+\left.\mu V\right|_{1} u_{\mathrm{s}}+3 g u_{\mathrm{s}}\left(A^{2} v^{2}\right), \\
& \dot{A} v=\mathcal{L}_{u} \varphi_{(2)}
\end{aligned}
$$

denotes the derivative with respect to $\tau$. We can solve these equations for $u_{(2)}$ and $\varphi_{(2)}$ and the solutions are

$$
\begin{aligned}
& u_{(2)}(\tau, x)=\beta(\tau) w(x)-\mu s(x)-A^{2}(\tau) r(x), \\
& \varphi_{(2)}(\tau, x)=\dot{A}(\tau) \rho(x),
\end{aligned}
$$

where $\mathcal{L}_{\varphi} w=u_{\mathrm{s}}, \beta=\partial_{\tau} \alpha, \mathcal{L}_{\varphi} s=V \mid{ }_{1} u_{\mathrm{s}}, \mathcal{L}_{\varphi} r=3 g u_{\mathrm{s}} v^{2}$. w, $s$ and $r$ exist because

$$
\left\langle v, u_{\mathrm{s}}\right\rangle=0 \quad \text { and } \quad\left\langle v, u_{\mathrm{s}} v^{2}\right\rangle=0 \quad \text { and } \quad\left\langle v,\left.V\right|_{1} u_{\mathrm{s}}\right\rangle=0 \text {. }
$$

Moreover, the parities are $w \odot u_{\mathrm{s}}, s \odot u_{\mathrm{s}}$ and $r \odot u_{\mathrm{s}}$. The function $\rho(x)$ that verifies $\mathcal{L}_{u} \rho=v$ can be explicitly computed and is given by $\partial_{x} \rho=u_{\mathrm{s}}^{-2} \int_{-\infty}^{x} u_{\mathrm{s}} v \mathrm{~d} x . \partial_{x} \rho$ is even.

At $\operatorname{order} \epsilon^{3 / 2}$, we have

$u_{\mathrm{s}} \rho \ddot{A}+A \beta v=\mathcal{L}_{\varphi} u_{(3)}+\left.\mu V\right|_{1} A g+6 g u_{\mathrm{s}}(A v)\left(\beta w-\mu s-A^{2} r\right)+g(A v)^{3}$

$w \dot{\beta}-2 r A \dot{A}=\mathcal{L}_{u} \varphi_{(3)}+2 A \dot{A} \partial_{x} v \partial_{x} \rho+A \dot{A} v \partial_{x x}^{2} \rho$.

\footnotetext{
${ }^{3}$ Actually $v$ can be considered as an extension of the translational mode $\partial_{x} u_{\mathrm{s}} . v$ verifies $\mathcal{L}_{\varphi}\left(v-\partial_{x} u_{\mathrm{s}}\right)=\left.u_{\mathrm{s}} \partial_{x} V\right|_{1}$.
} 
To find $u_{(3)}$, we have to write the solvability condition

$$
\begin{gathered}
\ddot{A}\left\langle v, u_{\mathrm{s}} \rho\right\rangle+\mu A\left\langle v,-V \mid{ }_{1} v+6 g u_{\mathrm{s}} v s\right\rangle+A \beta\left(\left\langle v, v-6 g u_{\mathrm{s}} w v\right\rangle\right) \\
+A^{3}\left(-g\left\langle v, v^{3}\right\rangle+6 g\left\langle v, u_{\mathrm{s}} r v\right\rangle\right)=0 .
\end{gathered}
$$

The problem associated with $\varphi_{(3)}$ can be written formally as $\mathcal{L}_{u} \varphi_{(3)}=\zeta$, where

$$
\zeta=w \dot{\beta}-2 r A \dot{A}-2 A \dot{A} \partial_{x} v \partial_{x} \rho-A \dot{A} v \partial_{x x}^{2} \rho .
$$

The solution of this equation is given by $\partial_{x} \varphi_{(3)}=u_{\mathrm{s}}^{-2} \int_{-\infty}^{x} u_{\mathrm{s}} \zeta \mathrm{d} x$. A necessary condition to bound the energy is then $\int_{-\infty}^{+\infty} u_{\mathrm{s}} \zeta \mathrm{d} x=0$ which is written

$$
\dot{\beta}\left\langle u_{\mathrm{s}}, w\right\rangle+A \dot{A}\left\langle u_{\mathrm{s}},-2 r-2 \partial_{x} v \partial_{x} \rho-v \partial_{x x}^{2} \rho\right\rangle=0 .
$$

Equations (44) and (42) have the same form as equations (14) and (15). Equation (44) states that there is a conserved quantity while equation (42) describes a reversible pitchfork bifurcation. We finally make a change of variables (in appendix B) to get the classical equations describing a reversible pitchfork bifurcation with a conserved quantity

$$
\begin{aligned}
& \partial_{\tau} Q=0, \\
& \partial_{\tau \tau}^{2} X=\tilde{\mu} X+Q X-X^{3} .
\end{aligned}
$$

To understand the meaning of equations (45) and (46), it is necessary to have a physical meaning for the two variables $Q$ and $X$. As shown in appendix C, equation (45) states the conservation of mass. Consequently, $Q$ is simply the perturbation of the mass. Equation (46) is related to the conservation of energy (in a less direct way). We will therefore interpret $X$ as the perturbation of the position of the centre of mass of the condensate.

\subsection{The description of weakly open condensates}

Inside the condensate, recombination occurs through two- and three-body collisions [11]. This is a mechanism of loss of atoms in the condensate. Losses in condensates are not homogeneous. They are preferably located at the edge of the condensate. In order to feed the condensate a pumping process is needed taking atoms from the uncondensed part and injecting them into the condensate. This leads to a permanent non-reversible dynamics induced by the coupling between condensed atoms and their environment.

We still use a mean field description and introduce phenomenologically irreversible terms into the NLSE. The equation that we will use reads

$$
\mathrm{i} \partial_{t} \Psi=-\partial_{x x}^{2} \Psi+g|\Psi|^{2} \Psi+V(x) \Psi+\mathrm{i}\left(v_{i} \Psi-v_{l}|\Psi|^{2} \Psi+v_{d} \partial_{x x}^{2} \Psi\right),
$$

where $v_{i}$ measures the rate of injection, $v_{l}$ measures the effect of two-body recombination and $v_{d}$ takes into account the non-homogeneity of the dissipative processes as well as thermal diffusion. All these coefficients will be small because the characteristic times of dissipation remain weak compared to the frequency of the trap.

These irreversible effects set the mass of the condensate. For a given set $v_{i}, v_{l}$ and $v_{d}$, a stationary solution $\Psi=u_{\mathrm{s}} \mathrm{e}^{-\mathrm{i} \omega_{\mathrm{s}} t}$ can be found $\left(u_{\mathrm{s}}\right.$ is not real). One property of this stationary solution is that the mass of the condensate should not evolve in time. The equation $\partial_{t} \int_{-\infty}^{+\infty}|\Psi|^{2} \mathrm{~d} x=0$ leads to

$$
v_{i} \int_{-\infty}^{+\infty}|\Psi|^{2} \mathrm{~d} x-v_{l} \int_{-\infty}^{+\infty}|\Psi|^{4} \mathrm{~d} x-v_{d} \int_{-\infty}^{+\infty} \partial_{x} \bar{\Psi} \partial_{x} \Psi \mathrm{d} x=0
$$




\subsection{Quasi-reversible self-trapping}

We now study how the irreversible effects can modify the dynamics of the self-trapping bifurcation. We still use the same asymptotic approach, assuming that the dissipative terms $v_{i}, v_{l}$ and $v_{d}$ are of order $\sqrt{\epsilon}$. In this limit, one describes the dynamics when dissipation and injection compete with the physical effects that lead to the bifurcation.

We use the ansatz

$$
\begin{aligned}
& u(x, t)=\sqrt{\epsilon} A(\tau) v(x)+\epsilon u_{(2)}(x, \tau)+\epsilon^{3 / 2} u_{(3)}(x, \tau)+\cdots \\
& \varphi(x, t)=\sqrt{\epsilon} \alpha(\tau)+\sqrt{\epsilon} \widehat{\varphi_{(1)}}(x, \tau)+\epsilon \varphi_{(2)}(x, \tau)+\epsilon^{3 / 2} \varphi_{(3)}(x, \tau)+\cdots
\end{aligned}
$$

The term $\widehat{\varphi_{(1)}}$ is a perturbation due to irreversible effects.

At $\operatorname{order} 1$, we have the same equation for $u_{\mathrm{s}}$ as equation (30):

$$
\omega_{\mathrm{s}} u_{\mathrm{s}}=-\partial_{x x}^{2} u_{\mathrm{s}}+3 g u_{\mathrm{s}}^{3}+\left.V\right|_{0} u_{\mathrm{s}} .
$$

As stressed before, this equation possesses a one-parameter family of solutions $\left(\omega_{\mathrm{s}}, u_{\mathrm{s}}\right)$. In the reversible case, we fixed the mass in order to select one of the solutions. In the quasi-reversible case the mass will be fixed by the irreversible effects at order $\sqrt{\epsilon}$.

At order $\sqrt{\epsilon}$, we have

$$
\begin{aligned}
& 0=A \mathcal{L}_{\varphi} v, \\
& 0=\alpha \mathcal{L}_{u} 1+\mathcal{L}_{u} \widehat{\varphi_{(1)}}+v_{i} u_{\mathrm{s}}-v_{l} u_{\mathrm{s}}^{3}+v_{d} \partial_{x x}^{2} u_{\mathrm{s}} .
\end{aligned}
$$

Equation (52) is just the definition of $v$ but equation (53) has a solution if and only if

$$
v_{i}\left\langle u_{\mathrm{s}}, u_{\mathrm{s}}\right\rangle-v_{l}\left\langle u_{\mathrm{s}}, u_{\mathrm{s}}^{3}\right\rangle-v_{d}\left\langle u_{\mathrm{s}}, \partial_{x x}^{2} u_{\mathrm{s}}\right\rangle=0,
$$

which is the leading order in equation (48). The amplitude of the condensate is selected by the irreversible processes and $\left(\omega_{\mathrm{s}}, u_{\mathrm{s}}\right)$ is completely determined by equations $(51)$ and $(54)$. The quasi-reversible terms induce a perturbation of phase and we have

$$
\mathcal{L}_{u} \widehat{\varphi_{(1)}}=-v_{i} u_{\mathrm{s}}+v_{l} u_{\mathrm{s}}^{3}-v_{d} \partial_{x x}^{2} u_{\mathrm{s}}
$$

and $\widehat{\varphi_{(1)}}$ is given by $\partial_{x} \widehat{\varphi_{(1)}}=\left(1 / u_{\mathrm{s}}^{2}\right) \int_{-\infty}^{x} u_{\mathrm{s}}\left(-v_{i} u_{\mathrm{s}}+v_{l} u_{\mathrm{s}}^{3}-v_{d} \partial_{x x}^{2} u_{\mathrm{s}}\right) \mathrm{d} \xi$ and $\widehat{\varphi_{(1)}}$ is even.

At order $\epsilon$, we have the same equations as equations (35) and (36) with additional terms

$u_{\mathrm{s}} \partial_{\tau} \alpha=\mathcal{L}_{\varphi} u_{(2)}+\left.\mu V\right|_{1} u_{\mathrm{s}}+3 g u_{\mathrm{s}}\left(A^{2} v^{2}\right)+u_{\mathrm{s}}\left(\partial_{x} \widehat{\varphi_{(1)}}\right)^{2}+v_{d} \mathcal{L}_{u} \widehat{\varphi_{(1)}}$,

$\partial_{\tau} A v=\mathcal{L}_{u} \varphi_{(2)}+2 A \partial_{x} v \partial_{x} \widehat{\varphi_{(1)}}+A v \partial_{x x}^{2} \widehat{\varphi_{(1)}}+v_{i} A v-v_{l} 3 u_{\mathrm{s}}^{2} A v+v_{d} A \partial_{x x}^{2} v$

We can solve these equations for $u_{(2)}$ and $\varphi_{(2)}$. The solution of equation (56) exists because

$$
\left\langle v, u_{\mathrm{s}}\left(\partial_{x} \widehat{\varphi_{(1)}}\right)^{2}\right\rangle=0, \quad\left\langle v, \mathcal{L}_{u} \widehat{\varphi_{(1)}}\right\rangle=0
$$

and the solution of equation (57) exists because

$$
\left\langle u_{\mathrm{s}}, \partial_{x} v \partial_{x} \widehat{\varphi_{(1)}}+v \partial_{x x}^{2} \widehat{\varphi_{(1)}}\right\rangle=0, \quad\left\langle u_{\mathrm{s}}, u_{\mathrm{s}}^{2} v\right\rangle=0, \quad\left\langle u_{\mathrm{s}}, \partial_{x x}^{2} v\right\rangle=0
$$

The solutions are

$$
\begin{aligned}
& u_{(2)}(\tau, x)=\beta(\tau) w(x)-\mu u_{1}(x)-A^{2}(\tau) r(x)-\widehat{u_{(2)}}, \\
& \varphi_{(2)}(\tau, x)=\partial_{\tau} A(\tau) \rho(x)-A \widehat{\varphi_{(2)}},
\end{aligned}
$$

where $\mathcal{L}_{\varphi} \widehat{u_{(2)}}=u_{\mathrm{s}}\left(\partial_{x} \widehat{\varphi_{(1)}}\right)+v_{d} \mathcal{L}_{u} \widehat{\varphi_{(1)}}$ and $\mathcal{L}_{u} \widehat{\varphi_{(2)}}=2 \partial_{x} v \partial_{x} \widehat{\varphi_{(1)}}+v \partial_{x x}^{2} \widehat{\varphi_{(1)}}+v_{i} A v-v_{l} 3 u_{\mathrm{s}}^{2} A v+$ $v_{d} A \partial_{x x}^{2} v$. Moreover, the parity are $\widehat{u_{(2)}} \odot u_{\mathrm{s}}$ and $\widehat{\varphi_{(2)}}$ is odd.

At order $\epsilon^{3 / 2}$, we have

$$
\begin{aligned}
& u_{\mathrm{s}} \rho \partial_{\tau \tau}^{2} A-u_{\mathrm{s}} \partial_{\tau} A \widehat{\varphi_{(2)}}+A \beta v=\mathcal{L}_{\varphi} u_{(3)}+\left.\mu V\right|_{1} A v+g(A v)^{3}+A v\left(\partial_{x} \widehat{\varphi_{(1)}}\right)^{2}+6 g u_{\mathrm{s}}(A v)\left(\beta w-\mu u_{1}-A^{2} r-\widehat{u_{(2)}}\right) \\
& w \partial_{\tau} \beta-2 r A \partial_{\tau} A=\mathcal{L}_{u} \varphi_{(3)}+2 A \partial_{\tau} A \partial_{x} v \partial_{x} \rho+A \partial_{\tau} A v \partial_{x x}^{2} \rho \\
&+2 \partial_{x}\left(\beta w-\mu u_{1}-A^{2} r-\widehat{u_{(2)}}\right) \partial_{x} \widehat{\varphi_{(1)}}+2 A^{2} \partial_{x} v \partial_{x} \widehat{\varphi_{(2)}} \\
&+A^{2} v \partial_{x x}^{2} \widehat{\varphi_{(2)}}+\left(\beta w-\mu u_{1}-A^{2} r-\widehat{u_{(2)}}\right) \partial_{x x}^{2} \widehat{\varphi_{(2)}} .
\end{aligned}
$$


To find $u_{(3)}$ and $\varphi_{(3)}$, we have to write two solvability conditions. The one associated with $\varphi_{(3)}$ is

$$
\begin{aligned}
\partial_{\tau} \beta\left\langle u_{\mathrm{s}}, w\right\rangle+ & A \partial_{\tau} A\left\langle u_{\mathrm{s}},-2 r-2 \partial_{x} v \partial_{x} \rho-v \partial_{x x}^{2} \rho\right\rangle \\
= & A^{2}\left\langle u_{\mathrm{s}}, 2 \partial_{x} v \partial_{x} \widehat{\varphi_{(2)}}-2 \partial_{x} r \partial_{x} \widehat{\varphi_{(1)}}+v \partial_{x x}^{2} \widehat{\varphi_{(2)}}-r \partial_{x x}^{2} \widehat{\varphi_{(1)}}\right\rangle \\
& +\left\langle u_{\mathrm{s}},-2 \mu \partial_{x} u_{1} \partial_{x} \widehat{\varphi_{(1)}}-\mu u_{1} \partial_{x x}^{2} \widehat{\varphi_{(1)}}-2 \partial_{x} \widehat{u_{(2)}} \partial_{x} \widehat{\varphi_{(1)}}-\widehat{u_{(2)}} \partial_{x x}^{2} \widehat{\varphi_{(1)}}\right\rangle \\
& +\beta\left\langle u_{\mathrm{s}}, 2 \partial_{x} w \partial_{x} \widehat{\varphi_{(1)}}+w \partial_{x x}^{2} \varphi_{(1)}\right\rangle .
\end{aligned}
$$

This solvability condition includes more terms than equation (44). All the additional terms are due to the irreversible process.

The other solvability condition reads

$$
\begin{gathered}
\partial_{\tau \tau}^{2} A\left\langle v, u_{\mathrm{s}} \rho\right\rangle+ \\
\left.+\partial_{\tau} A\left\langle v,-u_{\mathrm{s}} \widehat{\varphi_{(2)}}\right\rangle+A\left\langle v,-\left.\mu V\right|_{1} v+6 \mu g u_{\mathrm{s}} v u_{1}-v\left(\partial_{x} \widehat{\varphi_{(1)}}\right)^{2}+6 g u_{\mathrm{s}} v \widehat{u_{(2)}}\right)\right\rangle \\
+A \beta\left\langle v, v-6 g u_{\mathrm{s}} w v\right\rangle+A^{3}\left\langle v,-g v^{3}+6 g u_{\mathrm{s}} r v\right\rangle=0 .
\end{gathered}
$$

With the change of variables given in appendix B, equations (64) and (65) can be written as

$$
\begin{aligned}
& \partial_{\xi \xi}^{2} X=(\tilde{\mu}+\hat{\mu}) X-X^{3}+Q X-v_{X} \partial_{\xi} X \\
& \partial_{\xi} Q=v_{Q}\left(Q_{0}-Q\right)-\eta X^{2} .
\end{aligned}
$$

These equations are the normal form describing the quasi-reversible bifurcation. It includes the reversible case $\left(\hat{\mu}=0, v_{X}=0, v_{Q}=0, \eta=0\right)$. The dissipative coefficients $v_{X}, v_{Q}$ and $\eta$ are positive.

We have performed numerical simulations of the original weakly non-reversible NLSE equation (47) and we show some typical results in figure 9. The system clearly exhibits Lorenzlike behaviour, with chaotic alternation of the centre of mass of the condensate between the left and right wells. For different parameter values (but in the same range), non-symmetric limit cycles, symmetric limit cycles, stable non-symmetric stationary solutions can be observed.

The behaviour is richer than in the reversible case and this is principally due to the coupling term $-\eta X^{2}$ of equation (67). When the symmetry breaking occurs, the condensate moves to one side. The coupling term $-\eta X^{2}$ then leads to a decrease in the mass of the condensate which renders the symmetric state as stable again.

\section{Discussion}

There are two candidates for manifesting the self-trapping bifurcation: the ground state of an attractive condensate and the dark soliton of a repulsive condensate. Despite its weaker stability [19], the attractive condensate would be a good candidate in which to observe the self-trapping bifurcation because the self-trapping then occurs on the ground state. A careful numerical study of the tri-dimensional GPE would be useful to determine whether the selftrapping bifurcation can occur before the collapse of the attractive condensate. In the limit of a thin cigar-shaped trap, this is obviously the case since there the collapse does not occur. The variation of the scattering length from positive to negative value would also be a very good way to observe the self-trapping bifurcation [5].

The intensity of the laser sheet which is related to $\mu$ in the equations is easily tunable. It is possible to approach the self-trapping bifurcation and then to experimentally study the weakly nonlinear behaviour described by the amplitude equations.

Although a complete and coherent theoretical description of irreversible effects in BECs is not yet available, within the assumption of quasi-reversibility (i.e. in the limit where the dissipative and forcing effects are supposed to be of the same order than the distance from the 

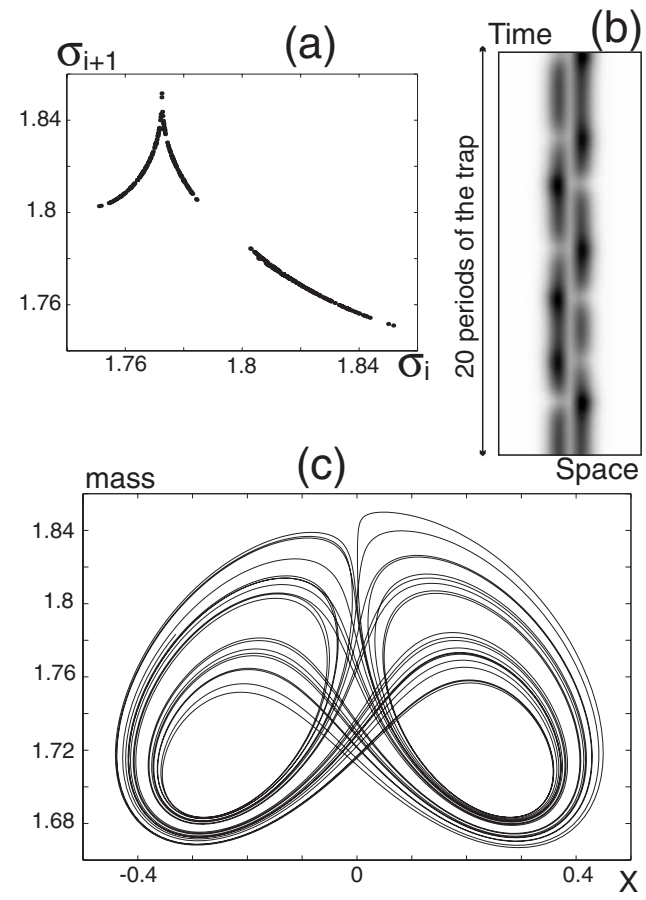

Figure 9. Numerical solutions of equation (47): $g=-1, b=6, c=10, \alpha=6, \beta=8, \gamma=3$ and $\epsilon=0.01$. (a) The plot of the successive maxima of the mass $\int|\Psi|^{2} \mathrm{~d} x$. (b) A density plot of $|\Psi|^{2}$ and $(c)$ is the phase space $\left(X=\int x|\Psi|^{2} \mathrm{~d} x\right)$.

instability threshold) the amplitude equation takes the same form whatever the microscopic modelling of the irreversible effects is. Irreversibility will always act as a damping of the mass $\left(v_{Q}\right)$, a damping of the motion of the centre of mass $\left(v_{X}\right)$ and stimulated mass losses $(\eta)$ will always occur. Only the numerical values of these parameters will be affected by the modelling. In our simulations, we used realistic values of the dissipative coefficients based on experimental observations [11]. As presented in [11], the values of the dissipative coefficient are strongly dependent on temperature. In any case, these coefficients remain small, such that the quasi-reversible approach is accurate for BECs.

The self-trapping bifurcation is a general feature of the competition between a potential bump and the nonlinearity. For example, when considering a repulsive BEC in a periodic trap, some solution with opposite phase in successive wells (which is an extension of the dark soliton) are found to be unstable [20].

\section{Summary}

We have discussed the dynamics of BECs in a double-well trap. The two-mode model has been used to describe the symmetry breaking self-trapping bifurcation in the reversible and the quasi-reversible cases. In this latter case we showed that the BEC can experience a transition to chaotic behaviours described by the Lorenz equations. We also used the one-dimensional NLSE to describe the self-trapping. In that case numerical experiments as well as the reduction to amplitude equations show that Lorenz-like chaotic behaviours can be observed. 


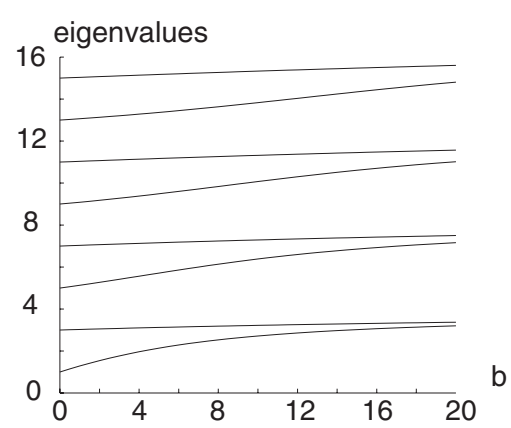

Figure A.1. The motion of the eigenvalues of the Schrödinger linear operator when $b$ evolves and some eigenmodes for different values of $b$ and $c=10$.

\section{Acknowledgments}

The authors wish to thank Professor E Spiegel for his useful suggestions on the paper. Numerical computations have been performed thanks to the NLKit software developed at the INLN.

\section{Appendix A. The weakly interacting limit: the two-mode model}

In this appendix we construct a low-dimensional model to describe the dynamics of a weakly interacting condensate in a double-well trap.

\section{A.1. The linear Schrödinger equation in a double-well trap}

We first recall some result on the linear Schrödinger equation

$$
\mathrm{i} \partial_{t} \Psi=-\partial_{x x}^{2} \Psi+V(x) \Psi .
$$

We will only consider the case where the potential is twice differentiable and the property $V( \pm \infty)=+\infty$ holds.

The stationary solutions are $\Psi=A \mathrm{e}^{-\mathrm{i} \Omega_{n} t} u_{n}(x)$. The eigenmodes $u_{n}(x)$ and the associated eigenvalues $\Omega_{n}$ of the operator $L=-\partial_{x x}^{2}+V(x)$ are separated (this a consequence of the Sturm-Liouville theorem). In a harmonic potential $V(x)=x^{2}$, the eigenvalues are regularly spaced. In a double-well potential (for example, we will consider a potential of the form $\left.V(x)=x^{2}+b \mathrm{e}^{-c x^{2}}\right)$ the eigenvalues will not be regularly spaced, the eigenvalues move and they gather by pairs. For example the eigenvalue associated with the ground state gets closer to the one associated with the first excited state (figure A.1).

The two lowest eigenvalues come closer to each other than the other modes. This behaviour suggests that some of the dynamic of the linear and the weakly NLSE could be understood with a low-dimensional model describing the interaction between these two modes.

\section{A.2. The one-mode model}

Let consider $g \sim \epsilon$ to be a small parameter. A simple perturbation of the first mode reads

$$
\Psi=\left[A u_{1}(x)+\epsilon p_{1}(x)\right] \mathrm{e}^{-\mathrm{i} \omega_{1} t},
$$

where $u_{1}$ is the fundamental mode (or any other mode) of the linear Schrödinger equation and $A$ is the slowly varying amplitude. $u_{1}$ is chosen to be normalized, i.e. $\int_{-\infty}^{+\infty} u_{1}^{2} \mathrm{~d} x=1$. At order 
$\epsilon^{0}$ the equation is just $\omega_{1} u_{1}=\mathcal{L}_{l} u_{1}$, where $\mathcal{L}_{l}=-\partial_{x x}^{2}+V(x)$ is the linear operator associated with the linear Schrödinger equation. At order $\epsilon$ the equation is

$$
\mathrm{i} \partial_{\tau} A u_{1}+\omega_{1} p_{1}=\mathcal{L}_{l} p_{1}+g|A|^{2} A u_{1}^{3},
$$

where the dots and the solvability condition to have $p_{1}$ finite is

$$
\mathrm{i} \partial_{\tau} A=\tilde{g}|A|^{2} A
$$

where $\tilde{g}=g \int_{-\infty}^{+\infty} u_{1}^{4} \mathrm{~d} x$. This equation is the classical description of a (weakly) nonlinear oscillator which simply states that the frequency of oscillations is a function of the amplitude of oscillations.

\section{A.3. The two-mode model}

We use the same procedure to derive the two-mode model. We consider two modes whose frequencies are very close. Let $\omega_{1}$ and $\omega_{2}$ be the frequencies of $u_{1}$ and $u_{2}$ and we assume $\omega_{1}+\omega_{2}=2 \omega_{m}$ and $\omega_{2}-\omega_{1}=\delta$, where $\delta$ is small $(\delta \sim \epsilon) . u_{1}$ and $u_{2}$ are normalized. We have

$$
\begin{array}{lll}
\left(\omega_{1}-\mathcal{L}_{l}\right) u_{1}=0 & \text { or } & \mathcal{L}_{m} u_{1}=\delta u_{1}, \\
\left(\omega_{2}-\mathcal{L}_{l}\right) u_{2}=0 & \text { or } & \mathcal{L}_{m} u_{2}=-\delta u_{2},
\end{array}
$$

where $\mathcal{L}_{m}=\omega_{m}-\mathcal{L}_{l}$. We use the ansatz

$$
\Psi=\left[A_{1} u_{1}(x)+A_{2} u_{2}(x)+\epsilon p\right] \mathrm{e}^{-\mathrm{i} \omega_{m} t} .
$$

The substitution in the NLSE gives at order $\epsilon^{0}$

$$
\mathcal{L}_{m} u_{1}=0 \quad \text { and } \quad \mathcal{L}_{m} u_{2}=0,
$$

which is true at this order. At order $\epsilon$ the two solvability conditions are

$$
\begin{aligned}
& \mathrm{i} \partial_{\tau} A_{1}=-\delta A_{1}+g c_{40}\left|A_{1}\right|^{2} A_{1}+2 g c_{22} A_{1}\left|A_{2}\right|^{2}+g c_{22} \overline{A_{1}} A_{2}^{2}, \\
& \mathrm{i} \partial_{\tau} A_{2}=\delta A_{2}+g c_{04}\left|A_{2}\right|^{2} A_{2}+2 g c_{22}\left|A_{1}\right|^{2} A_{2}+g c_{22} A_{1}^{2} \overline{A_{2}},
\end{aligned}
$$

where $c_{40}=\int_{-\infty}^{+\infty} u_{1}^{4} \mathrm{~d} x, c_{22}=\int_{-\infty}^{+\infty} u_{1}^{2} u_{2}^{2} \mathrm{~d} x$ and $c_{04}=\int_{-\infty}^{+\infty} u_{2}^{4} \mathrm{~d} x$.

Moreover, $c_{22}-c_{04} \sim \epsilon$ and $c_{04}-c_{40} \sim \epsilon$ [25]. In the two previous equations we can write $\tilde{g}=g \frac{1}{4} c_{40}$ and we have

$$
\begin{aligned}
& \mathrm{i} \partial_{\tau} A_{1}=-\delta A_{1}+\frac{1}{4} \tilde{g}\left|A_{1}\right|^{2} A_{1}+2 \frac{1}{4} \tilde{g} A_{1}\left|A_{2}\right|^{2}+\frac{1}{4} \tilde{g} \overline{A_{1}} A_{2}^{2}, \\
& \mathrm{i} \partial_{\tau} A_{2}=\delta A_{2}+\frac{1}{4} \tilde{g}\left|A_{2}\right|^{2} A_{2}+2 \frac{1}{4} \tilde{g}\left|A_{1}\right|^{2} A_{2}+\frac{1}{4} \tilde{g} A_{1}^{2} \overline{A_{2}} .
\end{aligned}
$$

This can also be transformed using the two variables $2 S=A_{1}+A_{2}$ and $2 D=A_{2}-A_{1}$. After substitution in equations (A.11) and (A.12), we get

$$
\begin{aligned}
& \mathrm{i} \partial_{\tau} S=\tilde{g}|S|^{2} S+\delta D, \\
& \mathrm{i} \partial_{\tau} D=\tilde{g}|D|^{2} D+\delta S,
\end{aligned}
$$

which is the two-mode model. $S$ represents the amplitude in one well, whereas $D$ represents the amplitude in the other well. These two coupled equations describe the interaction between the two wells. It is an extension of the linear two-mode model of a two-level system.

The two-mode model catches the universal behaviour of two coupled nonlinear oscillators. For example, the oscillations of two pendula coupled by a torsional spring (figure A.2) are described by

$$
\begin{aligned}
& \ddot{\theta}_{1}+\sin \theta_{1}=\kappa\left(\theta_{2}-\theta_{1}\right), \\
& \ddot{\theta}_{2}+\sin \theta_{2}=\kappa\left(\theta_{1}-\theta_{2}\right),
\end{aligned}
$$




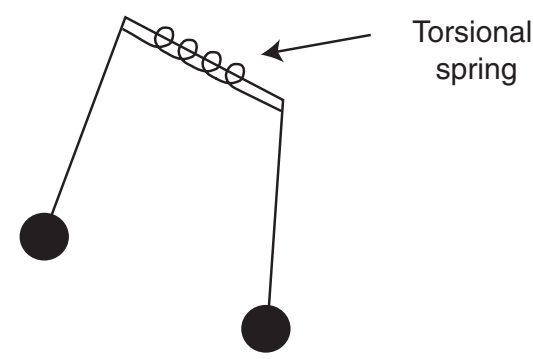

Figure A.2. Sketch of two coupled nonlinear oscillators: coupled pendula.

where $\kappa$ measures the intensity of the restoring torque due to the spring. The weak oscillations of the pendula can be caught by the asymptotic expansion

$$
\theta_{i}(t)=\sqrt{\epsilon}\left(A_{i}(T) \mathrm{e}^{-\mathrm{i} t}+\overline{A_{i}}(T) \mathrm{e}^{\mathrm{i} t}+\theta_{i}^{(3)}(t, T)+\cdots\right] .
$$

The solvability condition at order $\epsilon^{3 / 2}$, with $\kappa \sim \epsilon$ is

$$
\begin{aligned}
& \left.-2 \mathrm{i} A_{1}^{\prime}-\frac{1}{2} \mid A_{1}\right]^{2} A_{1}=\kappa\left(A_{2}-A_{1}\right), \\
& \left.-2 \mathrm{i} A_{2}^{\prime}-\frac{1}{2} \mid A_{2}\right]^{2} A_{2}=\kappa\left(A_{1}-A_{2}\right) .
\end{aligned}
$$

\section{Appendix B. The computation of the normal form}

In this appendix we transform equations (64) and (65) into adimensionalized equations. We work on the quasi-reversible equations because they include the reversible equations.

First we perform integration by parts to transform equation (64) into

$$
\begin{aligned}
\partial_{\tau} \beta\left\langle u_{\mathrm{s}}, w\right\rangle+ & A \partial_{\tau} A\left(-2\left\langle u_{\mathrm{s}}, r\right\rangle+\langle v, v\rangle\right)=\left(\mu\left\langle u_{1}, \widehat{\varphi_{(1)}}\right\rangle+\left\langle\widehat{u_{(2)}}, \widehat{\varphi_{(1)}}\right\rangle\right)-\beta\left\langle w, \widehat{\varphi_{(1)}}\right\rangle \\
& +A^{2}\left(-\left\langle v, \mathcal{L}_{u} \widehat{\varphi_{(2)}}\right\rangle+\left\langle r, \widehat{\mathcal{L}_{u} \widehat{\varphi_{(1)}}}\right\rangle\right) .
\end{aligned}
$$

Equation (65) can be presented as

$$
\begin{aligned}
\partial_{\tau \tau}^{2} A\left\langle v, u_{\mathrm{s}} \rho\right\rangle+ & \partial_{\tau} A\left\langle v,-u_{\mathrm{s}} \widehat{\varphi_{(2)}}\right\rangle-\mu A\left\langle v,\left.V\right|_{1} v-6 g u_{\mathrm{s}} v u_{1}\right\rangle-A\left\langle v, v\left(\partial_{x} \widehat{\varphi_{(1)}}\right)^{2}\right. \\
& \left.-6 g u_{\mathrm{s}} v \widehat{u_{(2)}}\right\rangle+A \beta\left\langle v, v-6 g u_{\mathrm{s}} w v\right\rangle+A^{3}\left\langle v,-g v^{3}+6 g u_{\mathrm{s}} r v\right\rangle=0 .
\end{aligned}
$$

Now, to get the adimensionalized equations (66) and (67), we use the change of variables

$$
Q=\frac{\left\langle v, v-6 g u_{\mathrm{s}} w v\right\rangle}{\left\langle v, u_{\mathrm{s}} \rho\right\rangle}\left[\beta+A^{2} \frac{1}{2\left\langle u_{\mathrm{s}}, w\right\rangle}\left(-2\left\langle u_{\mathrm{s}}, r\right\rangle+\langle v, v\rangle\right)\right] \text {, }
$$

$X=\| \frac{1}{\left\langle v, \rho u_{\mathrm{s}}\right\rangle}\left[-g\left\langle v, v^{3}\right\rangle+6 g\left\langle v, u_{\mathrm{s}} v r\right\rangle\right.$

$$
\left.-\frac{1}{2\left\langle u_{\mathrm{s}}, w\right\rangle}\left(-2\left\langle u_{\mathrm{s}}, r\right\rangle+\langle v, v\rangle\right)\left\langle v, v-6 g u_{\mathrm{s}} v w\right\rangle\right] \|^{1 / 2} A
$$

$\tilde{\mu}=\mu \frac{\left\langle v,\left.V\right|_{1} v-6 g u_{\mathrm{s}} v u_{1}\right\rangle}{\left\langle v, \rho u_{\mathrm{s}}\right\rangle}$,

$\hat{\mu}=\frac{\left\langle v, v\left(\partial_{x} \widehat{\varphi_{(1)}}\right)^{2}-6 g u_{\mathrm{s}} v \widehat{u_{(2)}}\right\rangle}{\left\langle v, \rho u_{\mathrm{s}}\right\rangle}$,

$v_{Q}=\frac{\left\langle w, \widehat{\varphi_{(1)}}\right\rangle}{\left\langle u_{\mathrm{s}}, w\right\rangle}$ 


$$
\begin{aligned}
& Q_{0}=\frac{\left(\mu\left\langle u_{1}, \widehat{\varphi_{(1)}}\right\rangle+\left\langle\widehat{u_{(2)}}, \widehat{\varphi_{(1)}}\right\rangle\right)\left\langle v, u_{\mathrm{s}} \rho\right\rangle}{\left\langle w, \widehat{\varphi_{(1)}}\right\rangle\left\langle v, v-6 g u_{\mathrm{s}} w v\right\rangle}, \\
& \eta=\left\{\frac{\left(\left\langle v, \mathcal{L}_{u} \widehat{\varphi_{(2)}}\right\rangle-\left\langle r, \widehat{\mathcal{L}_{u}} \widehat{\varphi_{(1)}}\right\rangle\right)\left\langle v, u_{\mathrm{s}} \rho\right\rangle}{\left\langle u_{\mathrm{s}}, w\right\rangle\left\langle v, v-6 g u_{\mathrm{s}} w v\right\rangle}-\frac{\left\langle w, \widehat{\varphi_{(1)}}\right\rangle\left(\langle v, v\rangle-2\left\langle u_{\mathrm{s}}, r\right\rangle\right)}{\left(\left\langle u_{\mathrm{s}}, w\right\rangle\right)^{2}}\right\} \\
& \quad \times\left\{\frac { 1 } { \langle v , \rho u _ { \mathrm { s } } \rangle } \left[-g\left\langle v, v^{3}\right\rangle+6 g\left\langle v, u_{\mathrm{s}} v r\right\rangle\right.\right. \\
& \left.\left.\quad-\frac{1}{2\left\langle u_{\mathrm{s}}, w\right\rangle}\left(-2\left\langle u_{\mathrm{s}}, r\right\rangle+\langle v, v\rangle\right)\left\langle v, v-6 g u_{\mathrm{s}} v w\right\rangle\right]\right\}^{-1}
\end{aligned}
$$

The sign of the expression under the absolute value in equation (B.4) is of prime interest. Depending on this value, the coefficient of the $X^{3}$ term in amplitude equation will be \pm 1 , i.e. the bifurcation will be supercritical or subcritical. Although we have not been able to find a general argument, it seems to be always positive for the potential chosen here.

\section{Appendix C. Conservation law}

There are many conserved quantities associated with the NLSE. Some of them can be deduced from the conserved quantities of the autonomous NLSE [21]. Two of them will be of particular interest, the mass

$$
M=\int_{-\infty}^{+\infty}|\Psi|^{2} \mathrm{~d} x
$$

and the energy

$$
E=\int_{-\infty}^{+\infty}\left(\left|\partial_{x} \Psi\right|^{2}+\frac{1}{2} g|\Psi|^{4}+V(x)|\Psi|^{2}\right) \mathrm{d} x
$$

An obvious question is whether or not these quantities are conserved by the set of the amplitude equations. Actually, we can use the ansatz of equations (27) and (28) to rebuild the set of two equations using the conservation laws for the mass and the energy. This provides a better physical interpretation for the amplitude equations.

\section{C.1. Conservation of mass}

We expect the conserved quantity $Q$ present in equation (45) to be associated with the mass. In equation (C.1) we have

$$
M=\int_{-\infty}^{+\infty}\left(u_{\mathrm{s}}+u\right)^{2} \mathrm{~d} x=M_{(0)}+\epsilon M_{(2)}+\epsilon^{2} M_{(4)},
$$

with

$$
\begin{aligned}
& M_{(0)}=\int_{-\infty}^{+\infty} u_{\mathrm{s}}^{2} \mathrm{~d} x \\
& M_{(2)}=\int_{-\infty}^{+\infty} 2 u_{\mathrm{s}}\left(\beta w-\mu u_{1}-A^{2} r\right)+A^{2} g^{2} \mathrm{~d} x \\
& M_{(4)}=\int_{-\infty}^{+\infty} 2 u_{\mathrm{s}} u_{(4)}+2 A v u_{(3)}+\left(\beta w-\mu u_{1}-A^{2} r\right)^{2} \mathrm{~d} x .
\end{aligned}
$$

Finally, equation (44) obtained by an asymptotic method is equivalent to what we get when we write $\partial_{t} M_{(2)}=0$. 


\section{C.2. Conservation of energy}

Now we expect equation (42) to be associated with the conservation of energy. In equation (C.2) we have

$$
\begin{gathered}
E=\int_{-\infty}^{+\infty}\left[\left(\partial_{x} u_{\mathrm{s}}+\partial_{x} u\right)^{2}+\left(\partial_{x} \phi\right)^{2}\left(u_{\mathrm{s}}+u\right)^{2}\right] \mathrm{d} x+\int_{-\infty}^{+\infty}\left[\frac{1}{2} g\left(u_{\mathrm{s}}+u\right)^{4}+V\left(u_{\mathrm{s}}+u\right)^{2}\right] \mathrm{d} x \\
=E_{(0)}+\epsilon E_{(2)}+\epsilon^{2} E_{(4)},
\end{gathered}
$$

with

$$
\begin{aligned}
E_{(0)}= & \int_{-\infty}^{+\infty}\left[\left(\partial_{x} u_{\mathrm{s}}\right)^{2}+\frac{1}{2} g u_{\mathrm{s}}^{4}+\left.V\right|_{0} u_{\mathrm{s}}^{2}\right] \mathrm{d} x \\
E_{(2)}= & \int_{-\infty}^{+\infty} 2 \partial_{x} u_{\mathrm{s}}\left(\left(\beta w-\mu u_{1}-A^{2} r\right)\right)+A^{2} v^{2} \mathrm{~d} x \\
& +\int_{-\infty}^{+\infty} \frac{1}{2} g\left[4 u_{\mathrm{s}}^{3}\left(\beta w-\mu u_{1}-A^{2} r\right)+6 u_{\mathrm{s}}^{2} A^{2} v^{2}\right] \mathrm{d} x \\
& +\left.\int_{-\infty}^{+\infty} V\right|_{0}\left[2 u_{\mathrm{s}}\left(\beta w-\mu u_{1}-A^{2} r\right)+A^{2} v^{2}\right]+\left.V\right|_{1} u_{\mathrm{s}}^{2} \mathrm{~d} x \\
= & \omega_{\mathrm{s}} M_{(2)} .
\end{aligned}
$$

If the mass is conserved (at order $\epsilon$ ) then the energy is also conserved:

$$
\begin{aligned}
& E_{(4)}=\int_{-\infty}^{+\infty} 2 \partial_{x} u_{\mathrm{s}} \partial_{x} u_{(4)}+2 A \partial_{x} g \partial_{x} u_{(3)} \mathrm{d} x \\
&+\int_{-\infty}^{+\infty}\left(\mu \partial_{x} u_{(1)}+A^{2} \partial_{x} r+\phi_{0} \beta \partial_{x} h\right)^{2}+\left(\partial_{\tau} A\right)^{2} \rho^{2} u_{\mathrm{s}}^{2} \mathrm{~d} x \\
&+\int_{-\infty}^{+\infty} \frac{1}{2} g\left[4 u_{\mathrm{s}}^{3} u_{(4)}+6 u_{\mathrm{s}}^{2}\left(\left(\mu u_{(1)}+A^{2} r+\phi_{0} \beta h\right)^{2}+2 A g u_{(3)}\right)\right] \mathrm{d} x \\
&+\int_{-\infty}^{+\infty} \frac{1}{2} g\left[4 u_{\mathrm{s}}\left(3 A^{2} g^{2}\right)\left(\mu u_{(1)}+A^{2} r+\phi_{0} \beta h\right)\right] \mathrm{d} x \\
&+\left.\int_{-\infty}^{+\infty} V\right|_{0}\left[2 u_{\mathrm{s}} u_{(4)}+2 A g u_{(3)}+\left(\mu u_{(1)}+A^{2} r+\phi_{0} \beta h\right)^{2}\right] \mathrm{d} x \\
&+\left.\int_{-\infty}^{+\infty} V\right|_{1}\left[2 u_{\mathrm{s}}\left(\mu u_{(1)}+A^{2} r+\phi_{0} \beta h\right)+A^{2} g^{2}\right] \mathrm{d} x \\
&= \omega_{\mathrm{s}} M_{(4)}-\int_{-\infty}^{+\infty}\left(\mu u_{(1)}+A^{2} r+\phi_{0} \beta h\right) \mathcal{L}_{\varphi}\left(\mu u_{(1)}+A^{2} r+\phi_{0} \beta h\right) \mathrm{d} x \\
&+\int_{-\infty}^{+\infty}+\left(\mu \partial_{x} u_{(1)}+A^{2} \partial_{x} r+\phi_{0} \beta \partial_{x} h\right)^{2}+\left(\partial_{\tau} A\right)^{2} \rho^{2} u_{\mathrm{s}}^{2} \mathrm{~d} x .
\end{aligned}
$$

Finally, the combination of the conservation of mass and of conservation of energy at order $\epsilon^{4}$ implies equation (42).

\section{Appendix D. Dynamic of a soliton in a slowly varying potential}

In this appendix we perform a perturbation analysis based on the dynamics of a soliton of the NLSE without potential. We introduce the slowly varying potential as a perturbation of the equation. There have been a large number of works related to the dynamics of solitons in nearly integrable systems (see [22,23]). In this appendix we use the conserved quantities of the NLSE to deduce the dynamic of the soliton in the slowly varying potential. 
We write the NLSE equation in the form

$$
\mathrm{i} \partial_{t} \Psi+\partial_{x x}^{2} \Psi+2|\Psi|^{2} \Psi=\epsilon V(x) \Psi .
$$

A solution of the NLSE without potential is given by

$$
\Psi(x, t)=2 \eta \frac{\mathrm{e}^{-\mathrm{i} \varphi}}{\cosh z},
$$

where $\varphi=-4 \eta^{2} t+\phi_{0}$ and $z=2 \eta\left(x-x_{0}\right)$. In order to catch the dynamics of the soliton in the slowly varying potential we assume that $\eta, x_{0}$ are slowly varying functions of time.

The conservation of the mass $M=\int_{-\infty}^{+\infty}|\Psi|^{2} \mathrm{~d} x=4 \eta$ can then be presented as

$$
\partial_{\tau} \eta=0,
$$

where $\tau$ is the length timescale.

The Ehrenfest theorem (which holds for the NLSE [24]) can be presented as

$$
\partial_{\tau \tau}^{2} \int_{-\infty}^{+\infty} x|\Psi|^{2} \mathrm{~d} x=-\int_{-\infty}^{+\infty} V^{\prime}(x)|\Psi|^{2} \mathrm{~d} x .
$$

At leading order we get

$$
4 \eta \partial_{\tau \tau}^{2} x_{0}=-4 \eta^{2} \int_{-\infty}^{+\infty} V^{\prime}(x) \frac{1}{\cosh ^{2}\left[2 \eta\left(x-x_{0}\right)\right]} \mathrm{d} x .
$$

We approximate the potential with $V(x)=\alpha x^{2}+\beta x^{4}$. We then get the equation for the position of the soliton

$$
\partial_{\tau \tau}^{2} x_{0}=-\left(2 \alpha+12 \frac{\beta}{4 \eta^{2}} \int_{-\infty}^{\infty} \frac{z^{2}}{c h^{2} z} \mathrm{~d} z\right) x_{0}-4 \beta x_{0}^{3} .
$$

We first notice than for a harmonic potential, the soliton behaves like a harmonic oscillator. When the barrier is formed, i.e. $\alpha$ is negative, the central position in the trap loses stability whenever

$$
\alpha<-6 \frac{\beta}{4 \eta^{2}} \int_{-\infty}^{\infty} \frac{z^{2}}{c h^{2} z} \mathrm{~d} z
$$

For the values given in figure 7 this would lead to instability for $b>0.073$, whereas the numerical simulation predicts a value $b=10$.

\section{References}

[1] Bradley C, Sackett C, Tolett J and Hulet R 1995 Phys. Rev. Lett. 759

[2] Davis K, Mewes M, Andrews M, Van Druten N, Durfee D, Kurn D and Ketterle W 1995 Phys. Rev. Lett. 75 3969

[3] Anderson M, Ensher J, Matthews M, Wieman C and Cornell E 1995 Science 269198

[4] Stringari F, Giorgini S, Pitaevskii L and Stringari S 1999 Rev. Mod. Phys. 71463

[5] Cornish S, Claussen J, Roberts J, Cornell E and Wieman C 2000 Phys. Rev. Lett. 851795

[6] Burger S, Bongs K, Dettmer S, Ertmer W, Sengstock K, Sanpera A, Shlyapnikov G and Lewenstein M 1999 Phys. Rev. Lett. 835198

[7] Andrews M, Townsend C, Miesner H, and Durfee D 1997 Science 275637

[8] Scott A 1999 Nonlinear Science: Emergence and Dynamics of Coherent Structures (Oxford: Oxford University Press)

[9] Milburn G, Corney J, Wright E and Walls D 1997 Phys. Rev. A 554318

[10] Coullet P and Vandenberghe N 2001 Phys. Rev. E 64025202

[11] Stamper-Kurn D, Miesner H-J, Andrews M and Ketterle W 1998 Phys. Rev. Lett. 81500

[12] Lorenz E 1963 J. Atmos. Sci. 20130

[13] Smerzi A, Fantoni S, Giovanazzi S and Shenoy S 1997 Phys. Rev. Lett. 794950 
[14] Ostrovskaya E, Kivshar Y, Lisak M, Hall B, Cattani F and Anderson D 2000 Phys. Rev. A 61031601

[15] Feynman R, Leighton R and Sands M 1963 The Feynman Lectures on Physics vol 3 (Reading, MA: AddisonWesley)

[16] Raghavan S, Smerzi A, Fantoni S and Shenoy S 1999 Phys. Rev. A 59620

[17] R K A 1975 PhD Thesis MIT, Cambridge

[18] Ruprecht P, Holland M, Burnett K and Edwards M 1995 Phys. Rev. A 514704

[19] Huepe C, Métens S, Dewel G, Borckmans P and Brachet M 1999 Phys. Rev. Lett. 821616

[20] Bronski J, Carr L, Deconinck B and Kutz J 2001 Phys. Rev. Lett. 861402

[21] Zakharov V and Shabat A 1973 Sov. Phys.-JETP 37823

[22] Newell A 1985 Solitons in Mathematics and Physics (Philadelphia: SIAM)

[23] Kivshar Y and Malomed B 1989 Rev. Mod. Phys. 61763

[24] Hasse R 1982 Phys. Rev. A 25583

[25] This can be proved using the WKB method: Landau L D and Lifshitz E M 1959 Quantum Mechanics—Nonrelativistic Theory (Reading, MA: Addison-Wesley) 